\title{
Catalytic hydrocyanation of activated terminal alkynes
}

\author{
David Tejedor, ${ }^{*}$ [a] Samuel Delgado-Hernández, [a][b] Lucía Colella[a] and Fernando \\ García-Tellado*[a]
}

\begin{abstract}
A universal, practical and scalable organocatalytic hydrocyanation manifold to provide $\beta$-substituted acrylonitriles bearing an electron-withdrawing functionality has been implemented. The catalytic manifold operates under the reactivity generation principle "a good nucleophile generates a strong base", and it uses DABCO as catalyst, activated terminal alkynes as substrates and acetone cyanohydrin as the cyanide source. The acrylonitriles obtained as $E, Z$ mixtures are straightforwardly resolved by simple flash chromatography delivering the pure isomers in preparative amounts.
\end{abstract}

Organic nitriles constitute appreciated versatile building blocks in material science, natural product synthesis and pharmaceutical industry.[1] Furthermore, the nitrile group is a versatile precursor of different functional groups,[2] including carboxylic acid derivatives, aldehydes, ketones, and heterocycles.[3] Among the different methods to gain access to these building blocks, the direct addition of $\mathrm{HCN}$ to carbon-carbon multiple bond (hydrocyanation) occupies a privileged place (Scheme 1).[4] Due to the low nucleophilicity of $\mathrm{HCN}$, the hydrocyanation of alkynes usually requires the use of a transition-metal catalyst to conveniently generate the corresponding alkenyl nitriles.[5] In the case of non-activated terminal alkynes, the metal-catalyzed hydrocyanation has been well studied, and branched (Markovnikov)[5a] or linear (anti-Markovnikov)[5d] acrylonitrile derivatives can be selectively obtained by a judicious choice of the metal catalyst (Scheme 1A). Outside the scope of these protocols are terminal alkynes bearing electron-withdrawing groups (EWG) (i.e., activated terminal alkynes), which are not suitable substrates for the direct metal-catalyzed hydrocyanation. Surprisingly, an alternative and efficient hydrocyanation protocol for these alkynes has not yet been described. The only reported example is the addition of potassium cyanide to methyl propiolate (DMSO, $\left.45^{\circ} \mathrm{C}\right)$ to provide the corresponding methyl 3cyanoacrylate in very low yield ( $24 \%$ yield).[6] However, a number of stepwise protocols have been described to gain access to acrylonitriles bearing different EWGs at the $\beta$-position (Scheme 1Ba-e).[7] Although these protocols have provided sufficient amounts of these acrylonitrile units for synthetic studies, they present severe drawbacks for preparative use. The main concerns rely on their stepwise character, their difficulty for

[a] Dr. D. Tejedor, Mr. S. Delgado-Hernández, Mrs. Lucía Colella and F. García-Tellado

Instituto de Productos Naturales y Agrobiología

Consejo Superior de Investigaciones Científicas

Astrofísico Francisco Sánchez 3, 38206 La Laguna, Tenerife, Spain

E-mail: fgarcia@ipna.csic.es; dtejedor@ipna.csic.es

[b] Mr. S. Delgado-Hernández

Doctoral and Postgraduate School, Universidad de La Laguna.

Supporting information for this article is given via a link at the end of the document
A) Hydrocyanation of terminal alkynes: general methodologies

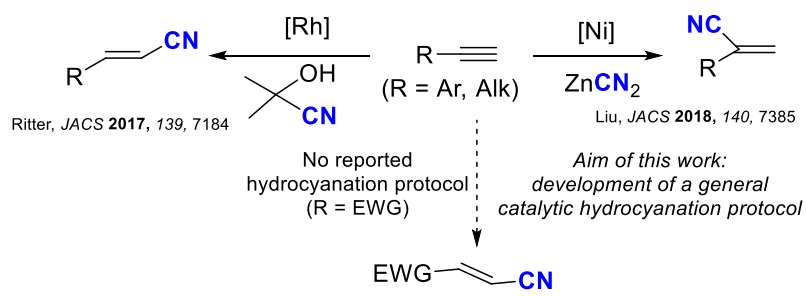

B) Stepwise generation of 3-EWG substituted acrylonitriles

a) 3-Cyano acrylates

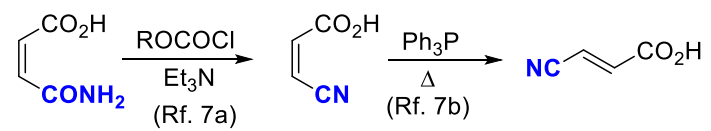

b) 3-Acyl acrylonitriles

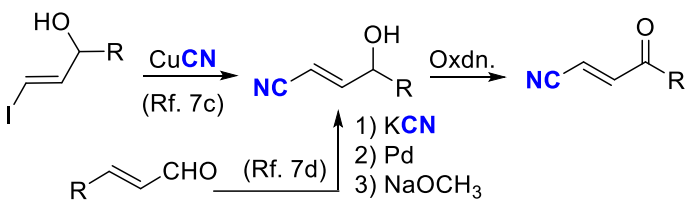

c) 3-Cyano acrylamides

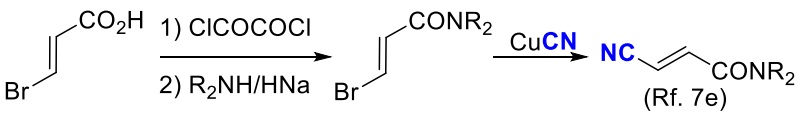

d) 3- Phosphonylacrylonitrile

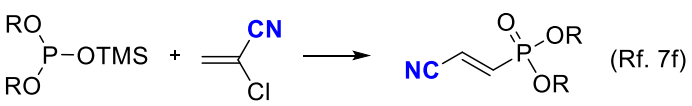

e) 3-Tosylacrylonitrile

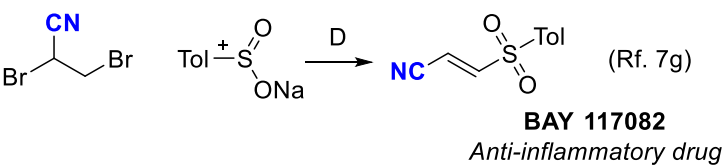

Scheme 1. A) Hydrocyanation of terminal alkynes. B) Usual stepwise protocols for the synthesis of 3-EWG substituted acrylonitriles.

scaling up and the use of elaborated reactants and/or toxic cyanide reagents. Therefore, the hydrocyanation of activated terminal alkynes remains an unresolved challenge in preparative synthetic chemistry, even in spite of the synthetic[8] and pharmaceutical[9] importance of the resulting 3-substituted acrylonitriles. Hydrocyanation protocols aimed to cover this gap with real-world preparative value should meet three main conditions for: 1) sustainability: they should be catalytic and scalable; 2) safety: the use or generation of toxic $\mathrm{HCN}$ should be avoided and 3) practicality: they should be universal, operationally simple, efficient and economic. With these principles as a guide, we have designed the catalytic manifold depicted in Scheme 2. 
The manifold is based on the reactivity generation principle a good nucleophile generates a strong base, $[10]$ and it uses an activated terminal alkyne as substrate, acetone cyanohydrin[11] as the cyanide source[5d] and 1,4-diazabicyclo[2.2.2]octane (DABCO) as the nucleophilic catalyst.[12] The catalytic cycle is initiated by the addition of DABCO onto the terminal alkyne to generate the zwitterion I, which should deprotonate the acetone cyanohydrin to generate the ammonium acrylate II and a catalytic amount of cyanide ion. The low nucleophilicity of the tertiary alkoxide guarantees the cyanide release. It was expected that the good matching between the nucleophilicity of the cyanide ion and the electrophilicity of the ammonium acrylate II would allow for a fast generation of the zwitterion III, which in turn, should deliver the acrylonitrile IV and DABCO to reinitiate the catalytic cycle. In terms of safety, only a catalytic amount of cyanide ion is generated and consumed in each catalytic cycle, precluding the accumulation of free cyanide ion[13] in the reaction medium. It must be noted that DABCO (pKa 8.7)[14] should not be basic enough to release cyanide from acetone cyanohydrin ( $\mathrm{pKa}$ 12.76).[15] In terms of chemical scope, the catalytic manifold is expected to be universal, that is, tolerant with a wide array of electron-withdrawing groups on the alkyne (i.e., ketones, esters, amides, phosphonates, sulphones, etc). As long as the terminal alkyne is properly polarized and its pKa remains higher than the pKa of the acetone cyanohydrin, the catalytic cycle should be fully operative. Herein, we report our results on the chemical design and successful implementation of this organocatalytic hydrocyanation manifold.

Catalytic principle:

a good nucleophile generates a strong base

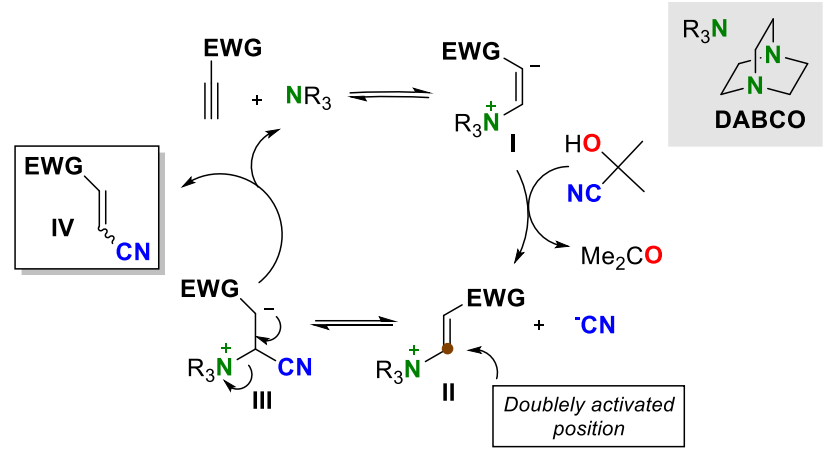

Scheme 2. Catalytic hydrocyanation manifold.

We undertook this work searching for the best set of experimental conditions to bring about the catalytic manifold, using the reaction of ethyl propiolate, acetone cyanohydrin and DABCO as the working model (Table 1). Gratifyingly, the reaction was highly efficient, affording $100 \%$ conversion under all the assayed conditions. Whereas the amount of DABCO did not influence the reaction efficiency (compare entries 1,11 and 12), it made a difference in the stereoselectivity of the reaction. The $E / Z$ ratio was doubled when the amount of DABCO was increased five times (entry 1 vs 12), but it did not vary when it was reduced one order of magnitude (entry 1 vs 11). Interestingly, the stereoselectivity of the catalytic reaction proved to be
Table 1. Optimization of the organocatalytic hydrocyanation manifold.

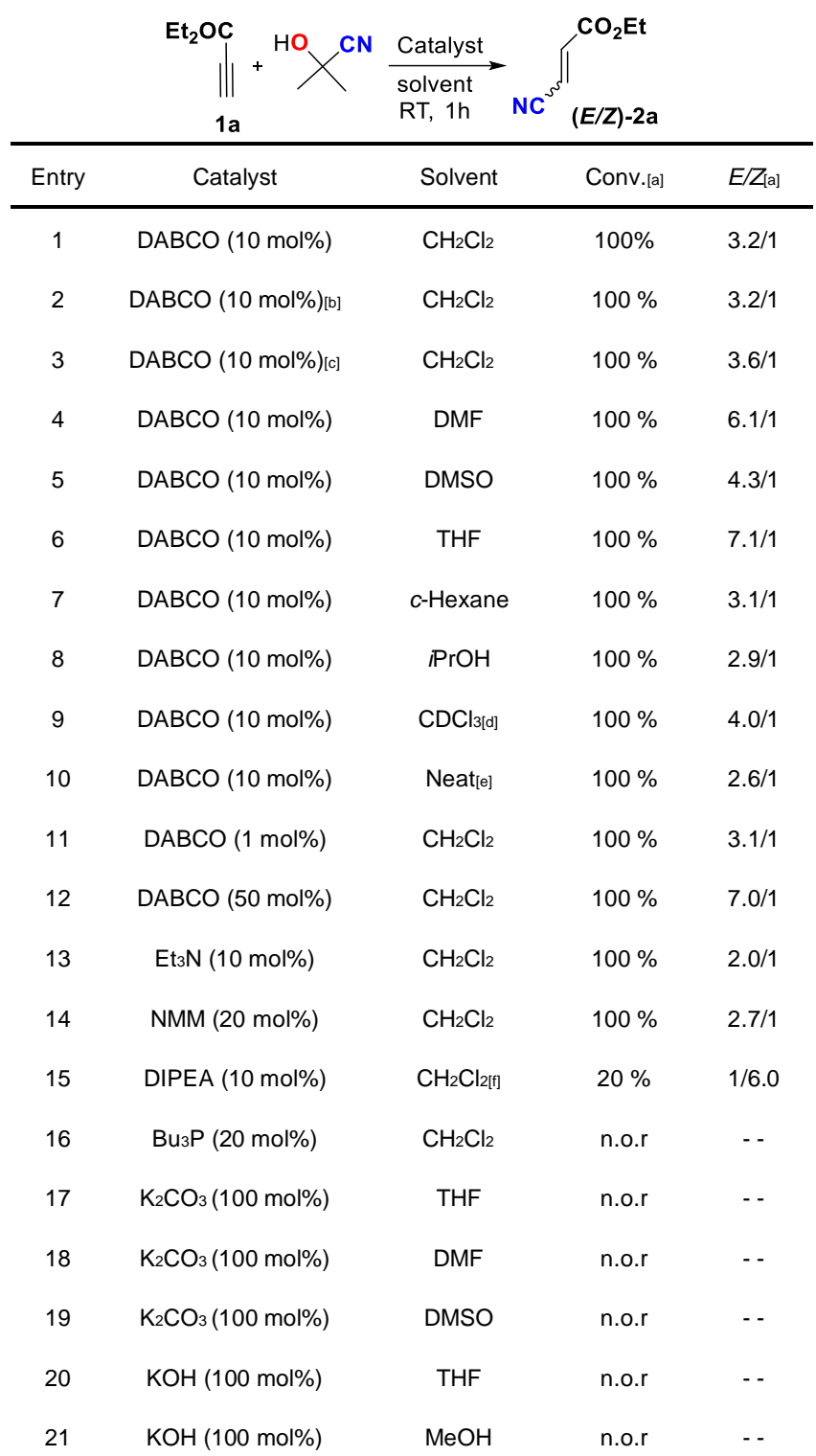

[a] By ${ }_{1} H$ NMR. [b] $0 \stackrel{\circ}{\circ} \mathrm{C}$. [c] $-78 \stackrel{\circ}{\circ}$. [d] Complete within the first 10 min. NMR tube. [e] Highly exothermic. [f] 3 hrs. [NMM $=N$-methylmorpholine. DIPEA $=N, N$-diisopropylethylamine. n.o.r. $=$ no observed reaction; product $2 \mathbf{a}$ not detected.

independent of the temperature (entries 1-3) but showed a subtle reliance with the solvent polarity (entries 3-9). The reaction could be performed in the absence of solvent, but under these conditions, it was highly exothermic and difficult to control (entry 10). The catalytic manifold also accepted other common Lewis bases as catalysts (entries 13-15), but none was as efficient as $\mathrm{DABCO}$, which could be used at very low charges (1 mol\%) without damaging the reaction efficiency (entry 11). DIPEA (pKa 10.75),[16] a worse nucleophile and a stronger base than DABCO (pKa 8.7), gave a much lower conversion (20\%, entry 15$)$, as 
should be expected for a Lewis base-catalyzed process (Scheme 2). The nucleophilic role of the catalyst was further confirmed by the inability of inorganic bases to catalyze the process (entries 1721). On the other hand, the low Michael-type reactivity of phosphonium acrylates[17] ruled out tertiary phosphines as suitable catalysts for these processes (entry 16). With all these results, we chose the conditions represented in entry 11 as the standard reaction conditions for the catalytic manifold.

With a standard set of experimental conditions in hand, we next explored the scope of the catalytic manifold with regard to the terminal alkyne (Table 2). As it can be observed, the catalytic manifold proved to be tolerant with a wide array of activating functionalities on the alkyne component. Satisfyingly, the chemical efficiency of the manifold was independent of the nature of the activating group (over $90 \%$ average yield), which confirmed its universal character. Only the more reluctant propiolamides 11p, ethynylidiphenylphosphine oxide (1q) and diethyl ethynylphosphonate (1r) called for a larger amount of DABCO (10 mol\%) and longer reaction times to get full conversions. In terms of stereoselectivity, although this was deeply marked by the own nature and substitution of the activating group, a predominant tendency to form the more stable $E$-isomer was observed in the majority of the cases. The only exception was found in the hydrocyanation of tosylacetylene (1s), which delivered the (Z)-3tosyl acrylonitrile (2s) as the major isomer,[18] allowing for the access to this hitherto inaccessible isomer in preparative amounts for biological/pharmacological studies. Although acrylonitriles 2a$\mathbf{r}$ were obtained as isomeric mixtures with $(E / Z)$ ratios spanning from $1.5 / 1$ to $50 / 1$, the preparative resolution of these mixtures was streamlined by the different molecular properties of both isomeric structures, i.e., symmetry, polarity and molar volume. TLC analysis of these mixtures consistently showed large elution differences for both geometrical isomers, which facilitated their straightforward separation by standard flash chromatography techniques. It is important to note that the simple and direct resolution of these mixtures allows providing both geometric isomers in preparative amounts, which minimizes the importance of stereoselectivity in these reactions. Other issues of particular practical importance are the simple reaction set up, the use of open atmospheres and simple reagents, and the formation of harmless byproducts (acetone).

Once the practical character of the catalytic manifold was demonstrated, we next studied its scalability, an intrinsic reaction value sought-after by the current sustainability paradigm in organic synthesis.[19] For this study, we chose the formation of the 3-cyanoacrylates $\mathbf{2 a}$ and $\mathbf{2 b}$ and the 3-cyanoacrylamide $\mathbf{2 m}$ because they are representative examples of highly demanded synthetic building blocks. As it can be observed in Table 2 (footnotes b-d), the three building blocks were synthesized on multigram scale, maintaining the standard reaction conditions, and without significant erosions in yields and/or stereoselectivities.

In summary, we have implemented a universal organocatalytic hydrocyanation manifold to provide $\beta$-substituted acrylonitriles bearing different electron-withdrawing functionalities (esters, ketones, amides, phosphonates, phosphine oxides and sulfones). The hydrocyanation manifold fills an important existent
Table 2. Scope of the organocatalytic hydrocyanation manifold.[a] Catalytic hydrocyanation
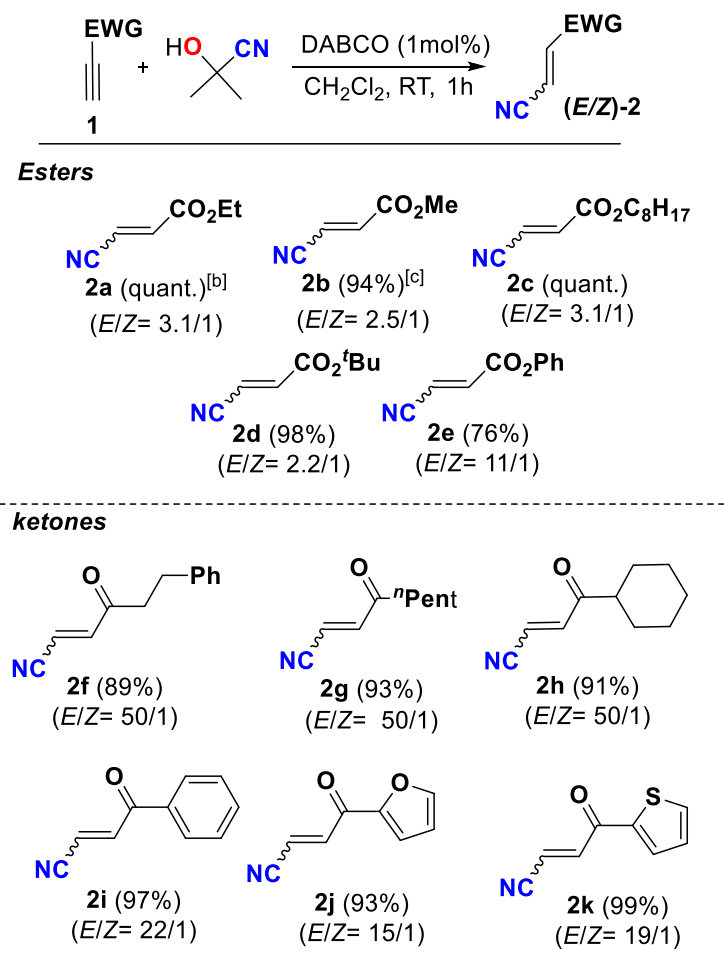

Amides (DABCO, $10 \mathrm{~mol} \%$ )
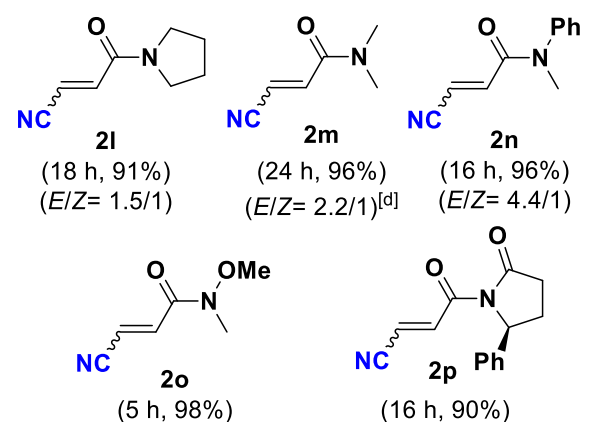

$(5 \mathrm{~h}, 98 \%)$

$(E / Z=6.3 / 1)$

$(16 \mathrm{~h}, 90 \%$ $E / Z=18 / 1)$

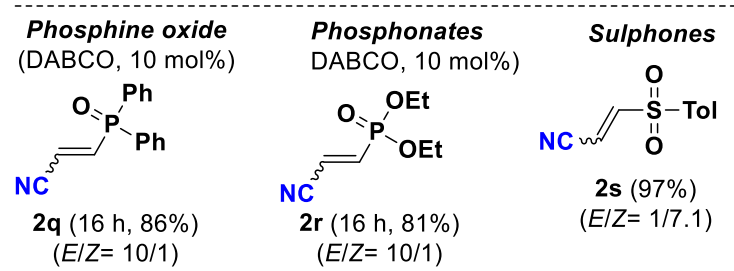

[a] The isomeric mixtures were resolved by flash chromatography. [b] The reaction performed at $50 \mathrm{mmol}$ scale gave $94 \%$ yield and a $E / Z$ ratio of $2.5: 1$.

[c] The reaction was performed at $10 \mathrm{mmol}$ scale. [d] The reaction performed at $18 \mathrm{mmol}$ scale gave $91 \%$ yield and a $E / Z$ ratio of $2.4: 1$.

methodological gap in the literature for accessing these deceptively simple molecules, which constitute highly valued building blocks in synthesis, medicinal chemistry and material sciences. Although the catalytic manifold delivers these structures as mixtures of $(E, Z)$-stereoisomers, their separation by standard 
flash chromatography techniques is simple and straightforward, providing the pure isomers in preparative amounts. We believe that the reported protocol offers a practical and unified solution for the hitherto unresolved direct synthesis of $\beta$-EWG substituted acrylonitriles from terminal alkynes, and we are confident that it will find widespread use in the preparative synthesis of these important building blocks.

\section{Experimental Section}

General procedure. Synthesis of (Z/E)-n-octyl 3cyanoacrylate (2c). A solution of $n$-octyl alkynoate $1 \mathrm{c}(363 \mathrm{mg}$; $2.0 \mathrm{mmol})$, acetone cyanohydrin (178.7 $\mathrm{mg} ; 2.1 \mathrm{mmol})$ and DABCO (2.2 mg; $0.02 \mathrm{mmol}$ ) in $\mathrm{CH}_{2} \mathrm{Cl}_{2}(4 \mathrm{~mL})$ was stirred for $1 \mathrm{~h}$ at room temperature. Solvent was evaporated under reduced pressure and the crude residue was flash chromatographed using silica gel. Elution with ethyl acetate-hexanes (10/90 v/v) afforded pure $(\boldsymbol{E})-2 \mathrm{c}(306.3 \mathrm{mg}, 73 \%)$. Colorless liquid: ${ }_{1} \mathrm{H}$ NMR $\left(\mathrm{CDCl}_{3}\right.$, $500 \mathrm{MHz}): \delta=0.85(\mathrm{t}, 3 \mathrm{H}, 3 \mathrm{~J}(\mathrm{H}, \mathrm{H})=6.9 \mathrm{~Hz}), 1.22-1.35(\mathrm{~m}, 10 \mathrm{H})$, 1.63-1.69 (m, 2H), $4.20(\mathrm{t}, 2 \mathrm{H}, 3 \mathrm{~J}(\mathrm{H}, \mathrm{H})=6.7 \mathrm{~Hz}), 6.47(\mathrm{~d}, 1 \mathrm{H}$, $3 J(\mathrm{H}, \mathrm{H})=16.4 \mathrm{~Hz}), 6.70(\mathrm{~d}, 1 \mathrm{H}, 3 \mathrm{~J}(\mathrm{H}, \mathrm{H})=16.4 \mathrm{~Hz}) \cdot{ }_{13} \mathrm{C}$ NMR $\left(\mathrm{CDCl}_{3}, 100 \mathrm{MHz}\right): \delta=14.0,22.6,25.6,28.4,29.07$ (2C), 31.7, $66.2,112.8,115.3,139.9,163.3 \mathrm{ppm}$. HRMS $\left(\mathrm{ESI}_{+}\right): \mathrm{m} / \mathrm{z}[\mathrm{M}+\mathrm{Na}]_{+}$ calculated for $\mathrm{C}_{12} \mathrm{H}_{19} \mathrm{NO}_{2} 232.1313$, found 232.1319. Further elution (30/70 v/v) delivered pure (Z)-2c (114.1 mg, 27\%). Colorless liquid: ${ }_{1} \mathrm{H}$ NMR $\left(\mathrm{CDCl}_{3}, 400 \mathrm{MHz}\right): \delta=0.86(\mathrm{t}, 3 \mathrm{H}$, $3 J(\mathrm{H}, \mathrm{H})=6.9 \mathrm{~Hz}), 1.25-1.38(\mathrm{~m}, 10 \mathrm{H}), 1.67-1.72(\mathrm{~m}, 2 \mathrm{H}), 4.24(\mathrm{t}$, $2 \mathrm{H}, 3 \mathrm{~J}(\mathrm{H}, \mathrm{H})=6.7 \mathrm{~Hz}), 5.93(\mathrm{~d}, 1 \mathrm{H}, 3 \mathrm{~J}(\mathrm{H}, \mathrm{H})=11.4 \mathrm{~Hz}), 6.70(\mathrm{~d}$, $1 \mathrm{H}, 3 \mathrm{~J}(\mathrm{H}, \mathrm{H})=11.4 \mathrm{~Hz}) .{ }_{13} \mathrm{C} \mathrm{NMR}\left(\mathrm{CDCl}_{3}, 100 \mathrm{MHz}\right): \delta=14.0$, 22.6, 25.8, 28.3, 29.07 (2C), 31.7, 66.1, 110.4, 114.2, 138.4, $162.5 \mathrm{ppm}$. HRMS $\left(\mathrm{ESI}_{+}\right): \mathrm{m} / \mathrm{z}[\mathrm{M}+\mathrm{Na}]_{+}$calculated for $\mathrm{C}_{12} \mathrm{H}_{19} \mathrm{NO}_{2}$ 232.1313 , found 232.1315 .

\section{Acknowledgements}

The authors thank the Spanish Ministries of Economy and Competitiveness (MINECO), Science, Innovation and Universities (MICINN), Agencia Estatal de Investigación (AEI) and the European Regional Development Funds (ERDF) for financial support (CTQ2015-63894-P and PGC2018-094503-B-C21). S. D. $\mathrm{H}$. thanks La Laguna University and Cajasiete for a pre-doctoral contract.

Keywords: Hydrocyanation - Terminal alkynes • DABCO • Organocatalysis $\cdot$ acetone cyanohydrin

[1] P. Pollak, G. Romeder, F. Hagedorn, H. Gelbke. Nitriles. In Ullman's Encyclopedia of Industrial Chemistry, 5th ed.; Wiley-VCH: Weinheim, Germany, 1985; Vol. A17, p 363.

[2] R. C. Larcok. Comprehensive Organic Transformations: A Guide to Functional Group Preparations, 2nd ed.; VCH: New York, 1999.

[3] M. Alterman, A. Hallberg, J. Org. Chem. 2000, 65, 7984-7989 and references cited therein.

[4] T. V. RajanBabu. Hydrocyanation in organic synthesis. In Comprehensive Organic Synthesis 2nd Eddition (Eds.: G. A. Molander, P. Knochel), Elsevier: Oxford, 2014, Vol. 5, pp 1772-1793.

[5] For selected recent examples, see: a) X. Zhang, X. Xie, Y. Liu, J. Am. Chem. Soc. 2018, 140, 7385-7389; b) A. Bhunia, K. Bergander, A. Studer,
J. Am. Chem. Soc. 2018, 140, 16353-1635; c) M. Shibuya, M. Okamoto, S. Fujita, M. Abe, Y. Yamamoto, ACS Catal. 2018, 8, 4189-419; d) F. Ye J. Chen, T. Ritter, J. Am. Chem. Soc. 2017, 139, 7184-7187; e) X. Fang P. Yu, G. P. Cerai, B. Morandi, Chem. Eur. J. 2016, 22, 15629-15633; f) S. Arai, Y. Amako, X. Yang, A. Nishida, Angew. Chem. Int. Ed. 2013, 52 8147-8150; Angew. Chem. 2013, 125, 8305-8308; g) T. Arai, Y. Suemitsu, Y. Ikematsu, Org. Lett. 2009, 11, 333-335.

[6] A Scifinder search by reaction, structure and topic only showed this result. W. Nagata, M. Yoshioka, Hydrocyanation of conjugated carbonyl compounds. In Organic Reactions, John Wiley \& Sons, Inc.: Hoboken, NJ 1977; Vol. 25, Chapter 3, pp 255; b) the $\alpha$-addition of cyanide to internal conjugated alkynes using a tertiary phosphine and acetone cyanohydrin has been described. A. Siby, O. Loreau, F. Taran, Synthesis 2009, 23652370 .

[7] a) C. K. Sauers, R. J. Cotter, J. Org. Chem. 1961, 26, 6-10; b) S Mukherjee, E. J. Corey, Org. Lett. 2010, 12, 1024-1027; c) I. Yamakawa H. Urabe, Y. Kobayashi, S. Fumie, Tetrahedorn Lett. 1991, 32, 2045 2048; d) A. Nudelman, E. Keinan, Synthesis 1982, 687-689; e) S. Harada T. Morikawa, A. Nishida, Org. Lett. 2013, 15, 5314-5317; f) K. Afarinkia M. Evans, J. C. H. Graham, G. Jimenez-Bueno, Tetrahedron Lett. 1998 , 38, 433-434; g) Z.-H. Guan, W. Zuo, L.-B. Zhao, Z.-H. Ren, Y.-M. Liang Synthesis 2007, 1465-1470.

[8] For recent selected examples, see: a) J.-H. Choia, C.-M. Park, Adv. Synth. Catal. 2018, 360, 3553-3562; b) Y. Liu, M. E. C. Nappi, P. Melchiorre, Org. Lett. 2012, 14, 1310-1313; c) references $7 \mathrm{~b}$ and $7 \mathrm{e}$.

[9] a) The (E)-3-tosylacrylonitrile, the so-called BAY 117082, is a potent antiinflammatory drug. S. Strickson, D. G.Campbell, C. H. Emmerich, A Knebel, L. Plater, M. S. Ritorto, N. Shpiro, P. Cohen, Biochem.J. 2013 $451,427-437$; b) for a review of nitrile-containing pharmaceuticals, see: $F$ F. Fleming, L. Yao, P. C. Ravikumar, L. Funk, B. C. Shook, J. Med. Chem. 2010, 53, 7902-7917.

[10] a) For the development of catalytic multicomponent manifolds based on this reactivity generation principle, see: D. Tejedor, S. López-Tosco, F. Cruz-Acosta, G. Méndez-Abt, F. García-Tellado, Angew. Chem. Int. Ed. 2009, 48, 2090-2098; Angew. Chem. 2009, 121, 2124-2131; b) D.Tejedor, F. Garcla-Tellado, J. J. Marrero-Tellado, P. de Armas, Chem. Eur. J. 2003, 9, $3122-3131$; c) for its application in the $\beta$-hydroxylation of $\alpha, \beta$ unsaturated carbonyl compounds, see: I. C. Stewart, F. D. Toste, R. G. Bergman, J. Am. Chem. Soc. 2003, 125, 8696-8697.

[11] S. A. Haroutounian. Acetone Cyanohydrin. In Encyclopedia of Reagents for Organic Synthesis, John Wiley \& Sons, 2001.

[12] DABCO has shown to be an excellent nucleophilic catalyst for this kind of processes. P. de Armas, F. García-Tellado, J. J. Marrero-Tellado, D. Tejedor, M. A. Maestro, J. González-Platas, Org. Lett. 2001, 3, 1905-1908.

[13] For toxicity issues, see: D. Udhayakumari, Sens. Actuators, B 2018 , 259,1022-1057.

[14] V. K. Aggarwal, I. Emme,. S. Y. Fulford, J. Org. Chem. 2003, 68, 692-700.

[15] https://chemaxon.com/products/calculators-and-predictors\#pka

[16] K. L. Sorgi. In Diisopropylethylamine, Encyclopedia of Reagents for Organic Synthesis, 2001. doi:10.1002/047084289X.rd254.

[17] a) D. Tejedor, D. González-Cruz, A. Santos-Expósito, J. J. MarreroTellado, P. de Armas, F. García-Tellado, Chem. Eur. J. 2005, 11, 35023510; b) D. Tejedor, A. Santos-Expósito, G. Méndez-Abt, C. Ruiz-Pérez, F. García-Tellado, Synlett, 2009, 1223-1226, c) B. M. Trost, G. R. Dake, J. Am. Chem. Soc. 1997, 119, 7595-7596.

[18] Z-predominance is commonly observed in nucleophilic additions on tosylacetylene. For a discussion, see: a) O. Arjona, R. Medel, J. Rojas, A. M. Costa, J. Vilarrasa, Tetrahedron Lett. 2003, 44, 6369-6373; b) J. S. Meek, J. S. Fowler, J. Org. Chem. 1968, 33, 985-991.

[19] C.-J. Li, B. M. Trost, Proc. Natl. Acad. Sci. 2008, 105, 13197-13202 and references cited therein. 
Entry for the Table of Contents (Please choose one layout)

\section{COMMUNICATION}

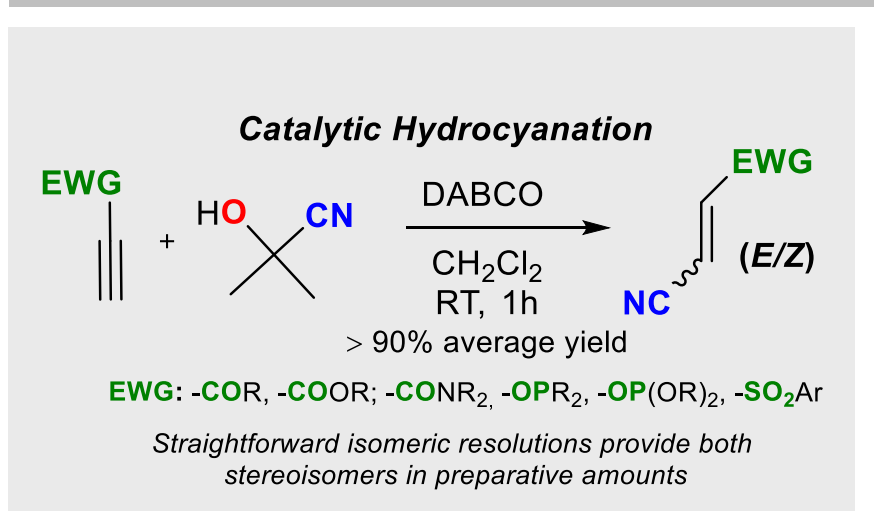

David Tejedor, * Samuel DelgadoHernández, Lucía Colella and Fernando García-Tellado*

Page No. - Page No.

Title: Catalytic hydrocyanation of activated terminal alkynes

Simplicity matters: A simple catalytic manifold using DABCO as catalyst and acetone cyanohydrin as the cyanide source performs the hydrocyanation of terminal activated alkynes with highly efficiency. The catalytic manifold operates under the reactivity principle a good nucleophile generates a strong base and tolerates a diverse array of activating groups on the alkyne. 